\title{
Sudden Transition from Equilibrium to Hybrid Chaos and Periodic Oscillations of the State-Dependent Round-Trip Delayed Nonsmooth Compound TCP with GRED Congestion Control System via HB-AFT
}

\author{
Lijun Pei $\mathbb{D}$, Hongyang Zhang $\mathbb{D}$, and Yueli Chen $(\mathbb{D}$ \\ School of Mathematics and Statistics, Zhengzhou University, Zhengzhou 450001, Henan, China \\ Correspondence should be addressed to Lijun Pei; peilijun99@163.com
}

Received 28 June 2020; Revised 30 September 2020; Accepted 2 November 2020; Published 21 November 2020

Academic Editor: Marcin Mrugalski

Copyright (c) 2020 Lijun Pei et al. This is an open access article distributed under the Creative Commons Attribution License, which permits unrestricted use, distribution, and reproduction in any medium, provided the original work is properly cited.

\begin{abstract}
In this paper, the nonsmooth compound transmission control protocol (TCP) with the gentle random early detection (GRED) system with the state-dependent round-trip delay is investigated in detail. Uniqueness of the positive equilibrium is proved firstly. Then, the closed approximate periodic solutions in this state-dependent delayed nonsmooth compound TCP with the GRED model are obtained by employing the harmonic balance and alternating frequency/time domain (HB-AFT) method. Then, we compare the results generated by numerical simulations with those of the closed approximate expressions obtained by HB-AFT. It indicates that HB-AFT is simple, correct, and powerful for state-dependent delayed nonsmooth dynamical systems. Finally, we find the complicated dynamic: chaos. It is a grazing chaos with a hybrid property, i.e., where usually $w$ oscillates at a very low frequency and $q$ oscillates at a very high frequency. And, the route to chaos is a very rare route, i.e., the instantaneous and local transition of stable equilibrium to chaos. So, to the end of stability and good performance, we should adjust the parameters carefully to avoid the periodic and chaotic oscillations.
\end{abstract}

\section{Introduction}

With the rapid growth of applications, network congestion is becoming more and more serious. At present, the whole congestion control system is a combination of the end-toend transmission control protocol (TCP) congestion control mechanism at the source and the active queue management $(\mathrm{AQM})$ mechanism at routers.

Although TCP can deliver data efficaciously in most applications, it is reported that TCP substantially cannot utilize successfully the network bandwidth over high-speed and long-distance networks [1]. Many approaches have been established to settle this problem. One class of these approaches is loss based, which uses packet loss as congestion feedback, including Tahoe TCP [2], HSTCP [1], STCP [3], and BIC-TCP [4]. Another class is delay based, which takes RTT variances as a congestion indicator, like FAST TCP [5].
However, some problems such as efficiency, RTT fairness, and TCP fairness cannot be concurrently mitigated either by loss-based or delay-based protocols. Compound TCP approach has been proposed in [6], which is a combination of delay-based and loss-based approaches. The approach has good efficiency, RTT fairness, and TCP fairness.

It is essential to consider queue management when solving the problem of network congestion. AQM has become a research hot spot because of the defects of passive queue management $(\mathrm{PQM})$. The main idea of $\mathrm{AQM}$ is to notify TCP sources to reduce their sending window to avoid congestion by marking or dropping packets before buffers fill up. Random early detection (RED), a typical AQM algorithm, was proposed in [7]. Floyd proposed gentle RED (GRED), the version of the RED algorithm, in [8]. Numerically congestion control systems operating on GRED may have a slight decrease in the amplitude of the oscillation, 
which indicates a positive effect on the system behaviors; however, there is an increase in the oscillation frequency in most cases [9]. Thus, it is important to consider the periodic solutions and complicated dynamics of the compound TCP with GRED congestion control systems in detail. There are various versions of AQM algorithms, such as random exponential marking (REM) [10] and proportional integral queue policy [11].

The stability and dynamics of compound TCP with the RED system are considered in some works. The authors studied the stability and bifurcation of compound TCP with the RED system in three different limiting regimes in $[12,13]$. In [14], chaotic phenomena and period doubling bifurcation of the first-order discrete time model for compound TCP with RED system are obtained by employing bifurcation diagrams and Lyapunov exponent. As far as we know, few works on the analytical approximate expression of the periodic oscillation of the state-dependent round-trip delayed and nonsmooth compound TCP-GRED system have been reported. As well known, the stability and performance of the network have close relationship with dynamics of the control system. So, it is significant to investigate their dynamics including periodic solution and chaos. Periodic solution is also the basis for the study of rich dynamics. The closed approximate periodic solution can help us adjust parameters to obtain the desired solutions. It is our motivation to research the rich dynamics and the closed approximate periodic solutions of the compound TCP with the GRED system.

Delay represents the tendency of some system counts not only on current but also on past historical states. It lays the basis of the delayed differential equation (DDE). So, it has important applications in the fields of physics, chemistry, information [15], neural networks [16, 17], biomathematics, finance [18], stock market, mechanics, aerospaces, electrical and circuits, and so on. Generally speaking, DDE exhibits more complicated dynamical behaviors than ordinary differential equations.

The harmonic balance method (HBM) is an efficient way to find the closed approximate periodic solutions in the nonlinear systems. Distinct methods are established from the classical HBM, such as the incremental harmonic balance (IHB) method [19, 20], the high-dimensional harmonic balance (HDHB) method $[21,22]$, the generalized harmonic balance (GHB) method [23], and the HB-AFT method [24-30]. In the process of solving the periodic solution, the HB-AFT method sets both the state variable and the nonlinear function as harmonic solution and establishes the relationship between harmonic coefficients according to the discrete time-frequency characteristics of the system. It does not need integration and series expansion for complex nonlinear terms. Therefore, the HB-AFT method is efficacious for the nonsmooth dynamical systems. In this paper, we derive the closed approximate periodic solutions for the state-dependent delayed nonsmooth compound TCP with GRED system by HB-AFT successfully.

We also explore the complicated dynamics of this system by numerical simulation. By the largest Lyapunov exponent and bifurcation diagram, we find (i) a grazing chaos with a hybrid property and (ii) the route to this chaos is the instantaneous and local transition of equilibrium to chaos. This phenomenon is very rare and has been reported in $[31,32]$. The direct transition from a stable equilibrium to quasiperiodicity in nonsmooth systems is also a rare phenomenon [33].

The remaining of this paper is organized as follows. We introduce the compound TCP with GRED model in the highspeed and long-distance networks in Section 2. We do not consider the average queue size in the computation of the packet-drop probability since it is not beneficial to stability of the model. The disadvantage of the model is that the router may not generate correct response when data streams enter the network suddenly. However, the model is relatively stable, which is excellent for the congestion control system. In Section 3, we briefly introduce the HB-AFT method. In Section 4, we present the analytical approximate expressions of the periodic solutions by HB-AFT and then we compare the results of numerical simulations with the analytical approximate expressions obtained by HB-AFT. The results show that the HB-AFT method is simple, correct, and efficient. Complicated and rich dynamics are presented in Section 5. The chaos is with hybrid states where $q$ has a very high frequency and a very small amplitude, but $w$ has a very low frequency and a large amplitude. The route to this chaos is a very rare sudden transition from equilibrium to chaos. Finally, we come to the conclusions of this paper in Section 6.

\section{Compound TCP-GRED Model}

In this section, we will present the compound TCP-GRED model. The standard TCP adjusts the data flow in the way of additive increase and multiplicative decrease, that is, the sending window size increases by one packet per round-trip time (RTT) and reduces to half of the current window size every time packet loss is detected. TCP variant increases the sending window by $i(w)$ per received acknowledgment and reduces it by $d(w)$ per packet loss. If a packet loss probability is $p(t)$, then it is acknowledged with probability $1-p(t)$. The average window size of TCP flow satisfies the following differential equation [34]:

$$
\dot{w}(t)=(i(w(t))(1-p(t))-d(w(t)) p(t-\tau)) \frac{w(t-\tau)}{\tau},
$$

where $\tau$ is the round-trip time, $w(t)$ is the average window size, and different TCP variants have different $i(w)$ and $d(w)$. Next, we will gain $i(w)$ and $d(w)$ for the compound TCP. The compound TCP adds a scalable delay-based component into the standard TCP; therefore, it is a combination of delay-based and loss-based approaches.

The congestion control algorithm of the compound TCP has two variables: cwnd, the congestion window; dwnd, the delay window. The sending window size follows that [6]

$$
\text { win }=\min (\text { cwnd }+ \text { dwnd, awnd }) \text {, }
$$

where awnd is the advertised window size. In the congestion avoidance stage, cwnd increases by one packet per RTT and 
halves every time packet loss is detected. The compound TCP sends cwnd + dwnd packets in one RTT. Therefore, when an acknowledgment is received, the congestion window is updated as

$$
\text { cwnd }=\frac{\text { cwnd }+1}{(\text { cwnd }+ \text { dwnd })} .
$$

The dwnd increases the window size when the network is underutilized and reduces it when congestion is detected. The algorithm of dwnd is designed as follows:

$$
\operatorname{dwnd}(t+1)= \begin{cases}\operatorname{dwnd}(t)+\left(\alpha \operatorname{win}(t)^{k}-1\right)^{+}, & \text {if diff }<\delta, \\ (\operatorname{dwnd}(t)-\varrho \operatorname{diff})^{+}, & \text {if diff } \geq \delta, \\ \left(\frac{\operatorname{win}(t)(1-\beta)-\text { cwnd }}{2}\right)^{+}, & \text {if loss, }\end{cases}
$$

where $\delta$ is a threshold for congestion detection, $\varrho$ defines how rapidly the delay window should reduce its window when early congestion is detected, parameters $\alpha, \beta$, and $k$ control the increase and decrease of the window size, $\alpha>0, \beta>0, k>0$, and parameter diff is the number of backlogged packets in the bottleneck queue, and it is estimated as

$$
\text { diff }=\left(\frac{\text { win }}{\text { baseRTT }}-\frac{\text { win }}{\text { RTT }}\right) \text { baseRTT, }
$$

where baseRTT is updated by the minimal RTT that has been observed so far when the connection is started. Therefore, the window size is updated as

$$
w(t+1)= \begin{cases}w(t)+\alpha w(t)^{k}, & \text { if no loss, } \\ w(t)(1-\beta), & \text { if loss. }\end{cases}
$$

We can get $i(w)=\alpha w^{k-1}$ and $d(w)=\beta w$ from (6).
Now, we consider dynamics of the queue size. Assuming there are $N$ end users, we let $W^{N}(t)$ be the sum of all the window sizes, and we can get the average window size $w(t)=W^{N}(t) / N$. Let $X^{N}(t)=W^{N}(t) / \tau$ be the rate at which packets arrive at the queue at time $t$, and the average arrival rate is $x(t)=X^{N}(t) / N$. So, $x(t)=w(t) / \tau$. If probability of packets loss is $p(t)$, then the packets would be queued with probability $1-p(t)$. Therefore, the total number of arrival queues is $(1-p(t)) \mu N x(t)$ in $\mu$ time. Let $C$ be the per-flow service capacity, and then the packets are served at a rate of $\widetilde{C}=$ NC. Therefore, in the time interval $[t, t+\mu]$, the queue size $Q^{N}(t)$ satisfies the following relationship [35]:

$$
Q^{N}(t+\mu) \approx\left[Q^{N}(t)+(1-p(t)) \mu N x(t)-\mu N C\right]_{0}^{B},
$$

where $[q]_{0}^{B}=\min (\max (q, 0), B)$ and $B$ is the size of the router buffer. Therefore, the queue size $q(t)=Q^{N}(t) / N$ satisfies the following differential equation:

$$
\dot{q}(t)= \begin{cases}(1-p(t)) \frac{w(t)}{\tau}-C, & \text { if } q(t)>0, \\ \max \left((1-p(t)) \frac{w(t)}{\tau}-C, 0\right), & \text { if } q(t)=0,\end{cases}
$$

where $p(t)$ is the probability of packet loss.

RED applies an exponentially weighted moving average scheme to calculate the average queue size. The average queue size $r(t) \leftarrow\left(1-W_{q}\right) r(t)+W_{q} q(t)$, where $q(t)$ describes the instantaneous queue size and $0 \leq W_{q} \leq 1$ represents the queue weighting parameter. Because large values of the queue weighting parameter $W_{q}$ can aid stability [13], we only consider the special case that $W_{q}=1$ in this paper, which means the average queue is not considered. So, in the gentle RED algorithm, the packet-drop probability is given by

$$
p(t)= \begin{cases}0, & q(t) \leq \underline{b}, \\ \frac{(q(t)-\underline{b}) \bar{p}}{\bar{b}-\underline{b}}, & \underline{b}<q(t)<\bar{b}, \\ \frac{(1-\bar{p})(q(t)-\bar{b})}{\bar{b}}+\bar{p}, \bar{b} \leq q(t)<2 \bar{b}, 1, q(t) \geq 2 \bar{b}, & \end{cases}
$$

where $\underline{b}, \bar{b}$, are $\bar{p}$ are the minimum threshold, maximum threshold, and maximum packet-drop probability, respectively.
The compound TCP with gentle RED system is modeled by the following delay differential equations from (1), (8), and (9) in [13]: 


$$
\left\{\begin{array}{l}
\dot{w}(t)=(i(w(t))(1-p(t-\tau(t)))-d(w(t)) p(t-\tau(t))) \frac{w(t-\tau(t))}{\tau(t)}, \\
\dot{q}(t)=(1-p(t)) \frac{w(t)}{\tau(t)}-C
\end{array}\right.
$$

where $w(t)$ is the average window size (average of packets sent by all windows in one round-trip time) at time $t, q(t)$ is the queue size at time $t, i(w(t))=\alpha w(t)^{k-1}$, and $d(w(t))=\beta w(t)$. Parameters $\alpha, \beta$, and $k$ control the increase and decrease of the window size, $\alpha>0, \beta>0, k>0$; $p(t)$ represents the packet-drop probability at time $t ; \tau$ characterizes the round-trip time, $\tau(t)=T_{1}+(q(t) / C)$, comprising the propagation delay and the queuing delay; $\tau(t)$ is dependent on the state variable $q$, so it is a statedependent delay; $C$ is the per-flow link capacity.

Theorem 1. There exists a unique positive equilibrium $E^{*}=$ $\left(w^{*}, q^{*}\right)$ in system (10) for $0<k<1$.

Proof. Let $E^{*}=\left(w^{*}, q^{*}\right)$ be the positive equilibrium of system (10), and then $E^{*}=\left(w^{*}, q^{*}\right)$ should satisfy the following conditions:

$$
\left\{\begin{array}{l}
\alpha w^{* k-1}\left(1-p^{*}\right)-\beta w^{*} p^{*}=0 \\
\left(1-p^{*}\right) \frac{w^{*}}{\tau^{*}}-C=0
\end{array}\right.
$$

where $\tau^{*}=T_{1}+\left(q^{*} / C\right), 0<k<1$,

$$
p^{*}= \begin{cases}0, & q^{*} \leq \underline{b}, \\ \frac{\left(q^{*}-\underline{b}\right) \bar{p}}{\bar{b}-\underline{b}}, & \underline{b}<q^{*}<\bar{b}, \\ \frac{(1-\bar{p})\left(q^{*}-\bar{b}\right)}{\bar{b}}+\bar{p}, \bar{b} \leq q^{*}<2 \bar{b}, 1, q^{*} \geq 2 \bar{b} . & \end{cases}
$$

When $q \leq \underline{b}$, i.e., $p=0$, there is no equilibrium. When $\underline{b}<q<\bar{b}$, i.e., $p=((q-\underline{b}) \bar{p} / \bar{b}-\underline{b})$, we can obtain $w=\left(\mathrm{CT}_{1}+q / 1-p\right)$ from the second equation of equation (11); then substituting $w$ into the first equation, we can get the equation $\quad \alpha\left(\mathrm{CT}_{1}+q / 1-p\right)^{k-1}(1-p)-\beta\left(\mathrm{CT}_{1}+\right.$ $q / 1-p) p=0 . \quad$ Let $\quad F(q)=\alpha\left(\mathrm{CT}_{1}+q / 1-p\right)^{k-1}$ $(1-p)-\beta\left(\mathrm{CT}_{1}+q / 1-p\right) p$. Since $F^{\prime}(q)=(\beta \bar{p}(\underline{b}-q) / \bar{b}-$ $\underline{b}-\bar{p}(q-\underline{b}))+\left(\beta \bar{p}^{2}(\underline{b}-q)\left(q+\mathrm{CT}_{1}\right) /(\bar{b}-\underline{b}-\bar{p}(q-\underline{b}))^{2}\right)-$ $\left(\beta \bar{p}\left(q+\mathrm{CT}_{1}\right) / \bar{b}-\underline{b}-\bar{p}(q-\underline{b})\right)-(\alpha \bar{p} / \bar{b}-\underline{b})((\bar{b}-\underline{b})(q+$ $\left.\left.\mathrm{CT}_{1}\right) / \bar{b}-\underline{b}-\bar{p}(q-\underline{\underline{b}})\right)^{-1+k}+\left(\alpha(-1+k)\left(\bar{b}-\underline{b}+\bar{p}\left(\underline{b}+\mathrm{CT}_{1}\right)\right)\right.$ $/ \bar{b}-\underline{b}-\bar{p}(q-\underline{b}))\left((\overline{\bar{b}}-\underline{b})\left(q+\mathrm{CT}_{1}\right) / \bar{b}-\underline{b}-\bar{p}(q-\underline{b})\right)^{-2+k}<$ $0, q \in[\underline{b}, \bar{b}], F(q)$ is monotonically decreasing in $[\underline{b}, \bar{b}]$. And, $F(\underline{b})=\alpha\left(\mathrm{CT}_{1}+q\right)^{k-1}>0$ and $F(\bar{b})=\left(\beta \bar{p}\left(\bar{b}+\mathrm{CT}_{1}\right) /-1+\bar{p}\right)$ $+\alpha\left(\bar{b}+\mathrm{CT}_{1}\right)\left(\bar{b}+C T_{1} / 1-\bar{p}\right)^{-2+k}$. When $\bar{b}<q<2 \bar{b}$, i.e., $p=((q-\bar{b})(1-\bar{p}) / \bar{b})+\bar{p}$, we can obtain $w=\left(\mathrm{CT}_{1}+\right.$ $q / 1-p)$ from its second equation; then substituting $w$ into its first equation, we can get the equation $\alpha\left(\mathrm{CT}_{1}+q / 1-p\right)^{k-1}(1-p)-\beta\left(\mathrm{CT}_{1}+q / 1-p\right) p=0$. Let $G(q)=\alpha\left(\mathrm{CT}_{1}+q / 1-p\right)^{k-1}(1-p)-\beta\left(\mathrm{CT}_{1}+q / 1-p\right) p$.

Since $\quad G^{\prime}(q)=(\beta(q-\bar{b}+\bar{p}(2 \bar{b}-q)) /(-1+\bar{p})(2 \bar{b}-q))-$ $\left(\beta\left(q+\mathrm{CT}_{1}\right) / 2 \bar{b}-q\right)+\left(\beta(q-\bar{b}+\bar{p}(2 \bar{b}-q))\left(q+\mathrm{CT}_{1}\right) /\right.$ $\left.(-1+\bar{p})(-2 \bar{b}+q)^{2}\right)+\left(\alpha(-1+k)\left(2 \bar{b}+\mathrm{CT}_{1}\right) / 2 \bar{b}-q\right)$ $\left(\bar{b}\left(q+\mathrm{CT}_{1}\right) /(1-\bar{p})(2 \bar{b}-q)\right)^{-2+k}+(\alpha(-1+\bar{p}) / \bar{b})(\bar{b}(q+$ $\left.\left.\mathrm{CT}_{1}\right) /(1-\bar{p})(2 \bar{b}-q)\right)^{-1+k}<0, q \in[\bar{b}, 2 \bar{b}], G(q)$ is monotonically decreasing in $[\bar{b}, 2 \bar{b}]$. And, $G(\bar{b})=(\beta \bar{p}(\bar{b}+$ $\left.\left.\mathrm{CT}_{1}\right) /-1+\bar{p}\right)+\alpha\left(\bar{b}+\mathrm{CT}_{1}\right)\left(\bar{b}+\mathrm{CT}_{1} / 1-\bar{p}\right)^{-2+k}=F(\bar{b})$ and $\lim _{q \longrightarrow 2 \bar{b}^{-}} G(q)=\lim _{q \longrightarrow 2 \bar{b}^{-}}, p \longrightarrow 1^{-}-\beta\left(\mathrm{CT}_{1}+q / 1-p\right)=$ $-\infty$. When $q \geq 2 \bar{b}$, i.e., $p=1$, there is also no equilibrium. Therefore, if $G(\bar{b})=F(\bar{b})<0$, then there is only one equilibrium in $[\underline{b}, \bar{b}]$, but no equilibrium exists in $[\bar{b}, 2 \bar{b}]$. If $G(\bar{b})=F(\bar{b})>0$, then any equilibrium does not exist in $[\underline{b}, \bar{b}]$, but there is only one equilibrium in $[\bar{b}, 2 \bar{b}]$. After getting $q^{*}$, we can get $w^{*}$ according to its second equation. As mentioned previously, system (10) has the unique positive equilibrium $E^{*}=\left(w^{*}, q^{*}\right)$.

\section{HB-AFT Method}

We define $\widetilde{t}=\omega t$ and still use $t$ to denote $\widetilde{t}$, so equation (10) can be written as

$$
\left\{\begin{array}{l}
\omega \dot{w}(t)=\left(\alpha w(t)^{k-1}(1-p(t-\omega \tau(t)))-\beta w(t) p(t-\omega \tau(t))\right) \frac{w(t-\omega \tau(t))}{\tau(t)} \\
\omega \dot{q}(t)=(1-p(t)) \frac{w(t)}{\tau(t)}-C
\end{array}\right.
$$

where $\omega$ represents the frequency of periodic solution in system (10). Now, the period of periodic solution with frequency $w$ in system (10) becomes $2 \pi$. And, we define $f_{1}=$ $\left(\alpha w(t)^{k-1}(1-p(t-\omega \tau(t)))-\beta w(t) p(t-\omega \tau(t))\right)(w(t-$ $\omega \tau(t)) / \tau(t))$ and $f_{2}=(1-p(t))(w(t) / \tau(t))-C$. 
Next, we will use the HB-AFT method to obtain the closed approximate periodic solution in system (13). We carry out the harmonic balance process firstly. Because the period of periodic solution of system (13) is $2 \pi$, the state variables $w$ and $q$ in system (13) can be expressed as the following:

$$
\left(\begin{array}{c}
w(t) \\
q(t)
\end{array}\right)=\left(\begin{array}{c}
a_{w 0} \\
a_{q 0}
\end{array}\right)+\sum_{k=1}^{K}\left[\left(\begin{array}{c}
a_{w k} \\
a_{q k}
\end{array}\right) \cos (k t)-\left(\begin{array}{c}
b_{w k} \\
b_{q k}
\end{array}\right) \sin (k t)\right] .
$$

The nonlinear terms $f_{1}$ and $f_{2}$ can also be rewritten into

$$
\left(\begin{array}{l}
f_{1}(t) \\
f_{2}(t)
\end{array}\right)=\left(\begin{array}{c}
c_{w 0} \\
c_{q 0}
\end{array}\right)+\sum_{k=1}^{K}\left[\left(\begin{array}{c}
c_{w k} \\
c_{q k}
\end{array}\right) \cos (k t)-\left(\begin{array}{c}
d_{w k} \\
d_{q k}
\end{array}\right) \sin (k t)\right],
$$

where $K$ represents the largest number of harmonics.

Substituting expressions (14) and (15) into equation (13) and balancing each harmonic term, we can gain $4 K+2$ algebraic equations $g(P, Q)=0$, where

$$
\left(\begin{array}{l}
g(1) \\
g(2)
\end{array}\right)=\left(\begin{array}{l}
c_{w 0} \\
c_{q 0}
\end{array}\right)=0,
$$

with respect to the sine terms,

$$
\left(\begin{array}{c}
g(4 k-1) \\
g(4 k)
\end{array}\right)=-\omega k\left(\begin{array}{c}
a_{w k} \\
a_{q k}
\end{array}\right)+\left(\begin{array}{c}
d_{w k} \\
d_{q k}
\end{array}\right)=0,
$$

with respect to the cosine terms,

$$
\left(\begin{array}{c}
g(4 k+1) \\
g(4 k+2)
\end{array}\right)=-\omega k\left(\begin{array}{c}
b_{w k} \\
b_{q k}
\end{array}\right)-\left(\begin{array}{c}
c_{w k} \\
c_{q k}
\end{array}\right)=0,
$$

where $k=1,2, \ldots, K$.

$$
\left(\begin{array}{c}
P \\
Q
\end{array}\right)^{T}=\left(\begin{array}{ccccccccccc}
a_{w 0} & a_{q 0} & a_{w 1} & a_{q 1} & b_{w 1} & b_{q 1} & \ldots & a_{w K} & a_{q K} & b_{w K} & b_{q K} \\
c_{w 0} & c_{q 0} & c_{w 1} & c_{q 1} & d_{w 1} & d_{q 1} & \ldots & c_{w K} & c_{q K} & d_{w K} & d_{q K}
\end{array}\right)^{T} .
$$

In addition, let $P(4 K+3)=\omega$.

To gain the unknown coefficient $P$, we regard $Q$ as known. The algebraic equations (16a), (16b), and (16c) have $4 K+3$ unknown variables but only $4 K+2$ equations, so we exert the condition on the phase of the first harmonic of the periodic solution according to the fixed-phase method [21], i.e., $a_{w 1}=0$ or $b_{w 1}=0$ or $a_{q 1}=0$ or $b_{q 1}=0$. Therefore, we can get an algebraic equation $g(4 K+3)=0$.

In order to get the harmonic coefficient $P$ of state variables $w$ and $q$, we can use the iterative method to solve the algebraic equations $g=0$. We use the Newton-Raphson method in this paper, and the iterative formula is

$$
P^{j+1}=P^{j}-\left(J\left(P^{j}\right)\right)^{-1} g\left(P^{j}\right),
$$

where $J$ is the Jacobian matrix, that is,

$$
J=\frac{d g}{d P}=\frac{\partial g}{\partial P}+\frac{\partial g}{\partial Q} \frac{d Q}{d P}
$$

Noting that only $(d Q / d P)$ is unknown in (19), next we will establish the relationship between $Q$ and $P$ by the alternating frequency/time domain (AFT) technique.

For an initially given set of harmonic coefficient $P$, the discrete information of $w(t)$ and $q(t)$ in the time domain can be presented by the inverse discrete Fourier transform (IDFT), i.e.,

$$
\begin{aligned}
\left(\begin{array}{c}
w(n) \\
q(n)
\end{array}\right)= & \left(\begin{array}{c}
a_{w 0} \\
a_{q 0}
\end{array}\right)+\sum_{k=1}^{K}\left[\left(\begin{array}{c}
a_{w k} \\
a_{q k}
\end{array}\right) \cos \left(\frac{2 \pi k n}{N}\right)\right. \\
& \left.-\left(\begin{array}{c}
b_{w k} \\
b_{q k}
\end{array}\right) \sin \left(\frac{2 \pi k n}{N}\right)\right] .
\end{aligned}
$$

The corresponding discrete information of $w(t-\omega \tau(t))$ and $q(t-\omega \tau(t))$ in the time domain can be described as the following:

$$
\begin{aligned}
\left(\begin{array}{c}
w(n-\omega \tau(n)) \\
q(n-\omega \tau(n))
\end{array}\right)= & \left(\begin{array}{c}
a_{w 0} \\
a_{q 0}
\end{array}\right) \\
& +\sum_{k=1}^{K}\left[\left(\begin{array}{c}
a_{w k} \\
a_{q k}
\end{array}\right) \cos \left(\frac{2 \pi k n}{N}-k \omega \tau(n)\right)\right. \\
& \left.-\left(\begin{array}{c}
b_{w k} \\
b_{q k}
\end{array}\right) \sin \left(\frac{2 \pi k n}{N}-k \omega \tau(n)\right)\right],
\end{aligned}
$$

where

$n=0,1,2, \ldots, N-1, w(n), q(n), w(n-\omega \tau(n))$, and $q(n-$ $\omega \tau(n))$ are the values at the $n$th discrete time, and $N$ is the number of discrete time points.

According to (20) and (21), the discrete information of $f_{i}(w(t), q(t), w(t-\omega \tau(t)), q(t-\omega \tau(t)))(i=1,2)$ in the time domain can be expressed as the following:

$$
\left(\begin{array}{l}
f_{1}(n) \\
f_{2}(n)
\end{array}\right)=\left(\begin{array}{l}
f_{1}(w(n), q(n), w(n-\omega \tau(n)), q(n-\omega \tau(n))) \\
f_{2}(w(n), q(n), w(n-\omega \tau(n)), q(n-\omega \tau(n)))
\end{array}\right) .
$$

So, we can get $Q$ with $P$ as variables from (22) by the discrete Fourier transform (DFT), i.e.,

$$
\begin{aligned}
& \left(\begin{array}{c}
c_{w 0} \\
c_{q 0}
\end{array}\right)=\frac{1}{N} \sum_{n=0}^{N-1}\left(\begin{array}{c}
f_{1}(n) \\
f_{2}(n)
\end{array}\right), \\
& \left(\begin{array}{c}
c_{w k} \\
c_{y k}
\end{array}\right)=\frac{2}{N} \sum_{n=0}^{N-1}\left(\begin{array}{l}
f_{1}(n) \\
f_{2}(n)
\end{array}\right) \cos \left(\frac{2 \pi k n}{N}\right),
\end{aligned}
$$


TABLe 1: The procedure for the iteration of the fixed point $P$.

\begin{tabular}{l} 
(1) For a given initial value $P^{0}, g\left(P^{0}\right)$ and $J\left(P^{0}\right)$ can be obtained according to equations (16a), (16b), and (16c); (13)-(23a), (23b), and (23c) \\
(2) \\
We can get value of $P^{1}$ by iterating equation (18) \\
(3) \\
Repeat $(1)$ and (2) until the norm of $P^{j}-P^{j-1}$ is less than a specified precision $\epsilon$ \\
\hline
\end{tabular}

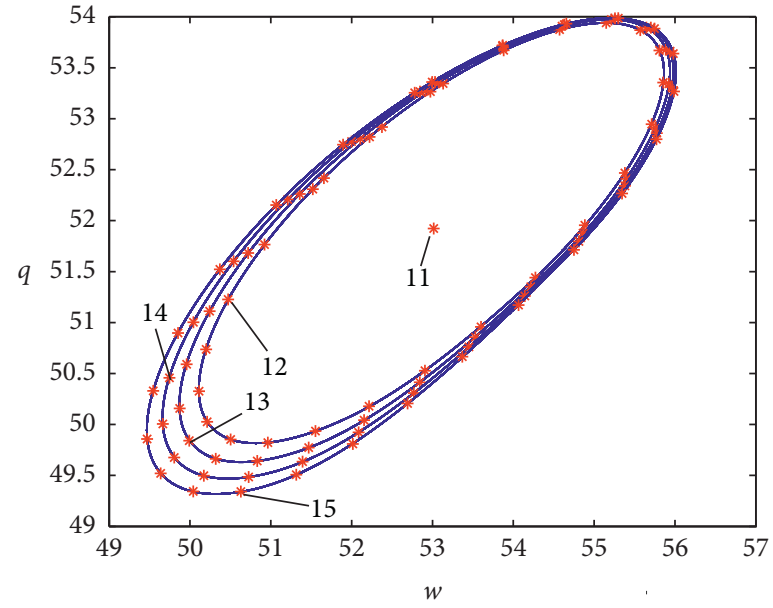

(a)

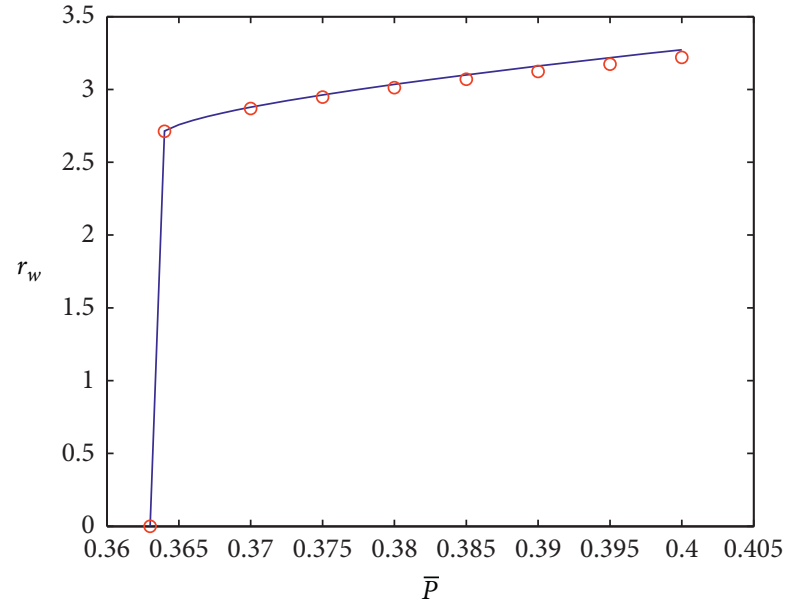

(b)

FIgURE 1: The phase portraits are displayed in (a) where solid blue lines are numerical results, ${ }^{*}$ results of the HB-AFT method, and $k=0.75, T_{1}=0.01, \alpha=0.125, \beta=0.5, C=100, \underline{b}=50$, and $\bar{b}=450 . l_{1}, l_{2}, l_{3}, l_{4}$, and $l_{5}$ correspond to $\bar{p}=0.363, \bar{p}=0.37, \bar{p}=0.38, \bar{p}=0.39$, and $\bar{p}=0.4$, respectively. The comparisons of amplitudes of $w$ in the numerical simulations and the first-order harmonic amplitudes of $w$ by HB-AFT are presented in (b) where the solid blue line is the amplitude of numerical simulations and red ${ }^{\circ}$ are the first-order harmonic amplitudes.

$$
\left(\begin{array}{l}
d_{w k} \\
d_{y k}
\end{array}\right)=-\frac{2}{N} \sum_{n=0}^{N-1}\left(\begin{array}{l}
f_{1}(n) \\
f_{2}(n)
\end{array}\right) \sin \left(\frac{2 \pi k n}{N}\right)
$$

where $k=1,2, \ldots, K$.

Now, we have obtained the relationship between $Q$ and $P$ from (20)-(23a), (23b), and (23c) by the AFT technique, so the expression of $(d Q / d P)$ can be obtained. Therefore, we can generate the harmonic coefficient $P$ by iterating formula (18), and then the closed approximate periodic solution can be obtained in system (13). Let $t \longrightarrow \omega t$, and we can obtain the closed approximate periodic solution in system (10). The procedure for the iteration of fixed point $P$ is briefly presented in Table 1.

\section{Numerical Simulations}

In this section, we will present the closed approximate periodic solutions of the compound TCP with GRED control system and perform the comparisons between results of the numerical simulations by WinPP and those of the HB-AFT method under different bifurcation parameters. The initial functions are $w(0)=q(0)=52$ and $w\left(-\tau_{\max }\right)=q\left(-\tau_{\max }\right)=$ 5 , and the step size is 0.0001 in numerical simulation in this section. The results are displayed in Figures $1-4$. We set the largest number of harmonics $K=5$ and the number of the discrete time points $N=144$ in the HB-AFT method in Figures 1-4. The results of the HB-AFT method are very consistent with those of numerical simulations as in Figures 1-4. So the accurate closed approximate periodic solutions can be obtained by HB-AFT for this state-dependent delayed nonsmooth system (10).

Moreover, since periodic oscillations can hinder network performance, we should avoid the periodic oscillations of system (10). It follows that the amplitudes of $w$ increase as parameter $\bar{p}$ increases in Figure 1, so we should select smaller $\bar{p}$ to avoid the periodic oscillations for $k=0.75, T_{1}=$ $0.01, \alpha=0.125, \beta=0.5, C=100, \underline{b}=50$, and $\bar{b}=450$. It follows that the amplitudes of $w$ increase as parameter $\underline{b}$ increases in Figure 2, so we should select smaller $\underline{b}$ to avoid the periodic oscillations for $k=0.75, T_{1}=0.01, \alpha=0.125, \beta=$ $0.5, C=100, \bar{p}=0.1$, and $\bar{b}=450$. Also, the amplitudes of $w$ increase as parameter $C$ increases in Figure 3, so we should select smaller $C$ to avoid the periodic oscillations for $k=$ $0.75, T_{1}=0.01, \alpha=0.125, \beta=0.5, \underline{b}=50, \bar{p}=0.35$, and $\bar{b}=$ 450. Also, the amplitudes of $w$ increase as parameter $T_{1}$ increases in Figure 4, so we should select smaller $T_{1}$ to avoid the periodic oscillations for $k=0.75, C=100, \alpha=0.125, \beta=$ $0.5, \underline{b}=50, \bar{p}=0.1$, and $\bar{b}=450$.

Now, we can obtain the closed approximate periodic solutions in system (10) by HB-AFT. Results of the closed approximate periodic solutions under three sets of parameters are shown in Table 2. For the first set of parameters, comparisons between results of numerical simulation and those of HB-AFT for system (10) are displayed in Figure 5. Obviously, the result obtained by the higher-order HB-AFT 


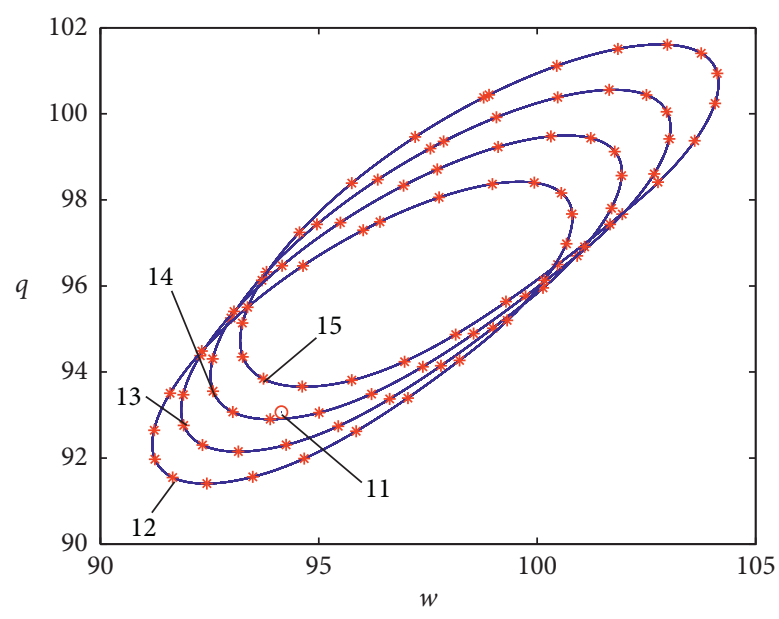

(a)

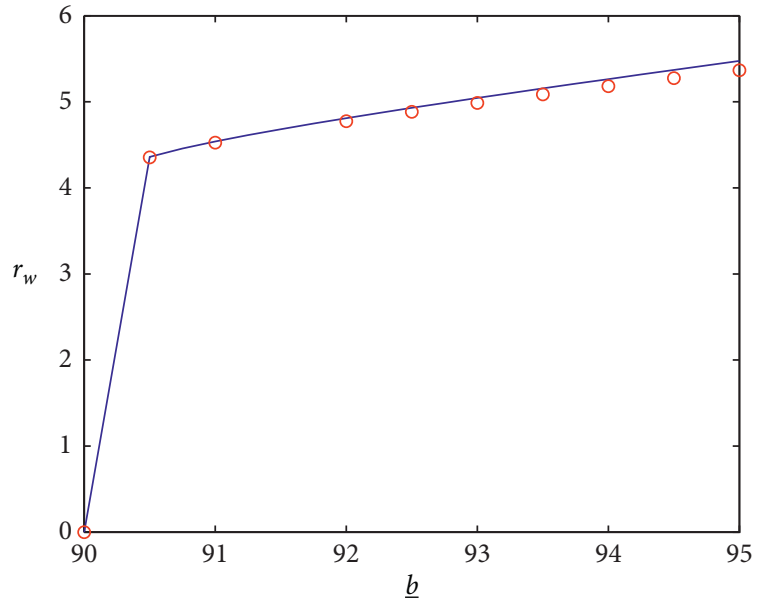

(b)

FIgURE 2: The phase portraits are displayed in (a) where solid blue lines are numerical results, ${ }^{*}$ and ${ }^{\circ}$ results of the HB-AFT method, and $k=0.75, T_{1}=0.01, \alpha=0.125, \beta=0.5, C=100, \bar{p}=0.1$, and $\bar{b}=450 . l_{1}, l_{2}, l_{3}, l_{4}$, and $l_{5}$ correspond to $\underline{b}=90, \underline{b}=92, \underline{b}=93, \underline{b}=94$, and $\underline{b}=95$, respectively. The comparisons of amplitudes of $w$ in the numerical simulations and the first-order harmonic amplitudes of $w$ by HBAFT are presented in (b) where the solid blue line is the amplitude of numerical simulations red ${ }^{\circ}$ are the first-order harmonic amplitudes.

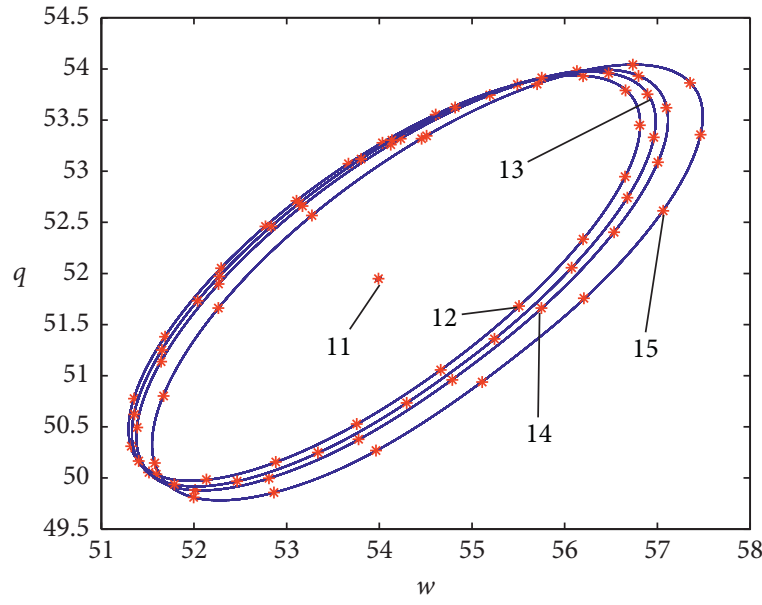

(a)



(b)

FIgUre 3: The phase portraits are displayed in (a) where solid blue lines are numerical results, ${ }^{*}$ results of the HB-AFT method, and $k=0.75, T_{1}=0.01, \alpha=0.125, \beta=0.5, \underline{b}=50, \bar{p}=0.35$, and $\bar{b}=450 . \quad l_{1}, l_{2}, l_{3}, l_{4}$, and $l_{5} \quad$ correspond $\quad$ to $C=195, C=199, C=$ $210, C=220$, and $C=250$, respectively. The comparisons of amplitudes of $w$ in the numerical simulations and the first-order harmonic amplitudes of $w$ by HB-AFT are presented in (b) where the solid blue line is the amplitude of numerical simulations and red ${ }^{\circ}$ are the firstorder harmonic amplitudes.

is better than that by the lower-order HB-AFT since it is in better agreement with the result of numerical simulation. Therefore, it is significant to select a proper number of harmonics in using HB-AFT.

For the second set of parameters, comparisons between the phase portraits of numerical simulations and those of the HB-AFT method are displayed in Figure 6(a).

For the third set of parameters, the closed approximate periodic solution is not shown here since it is too lengthy. The comparisons between the phase portrait of numerical simulation and that of the HB-AFT method are displayed in Figure 6(b). It follows that the accurate analytical approximate expression can be obtained even for the sharp impacting periodic solution by HB-AFT from Figure 6(b).

Therefore, the comparisons between the phase portrait of numerical simulation and that of the HB-AFT method in this section indicate that the HB-AFT is simple and efficient for the state-dependent delayed nonsmooth dynamical systems.

\section{Complicated Dynamics}

In this section, we study complicated dynamics of the statedependent round-trip delayed nonsmooth compound TCP 


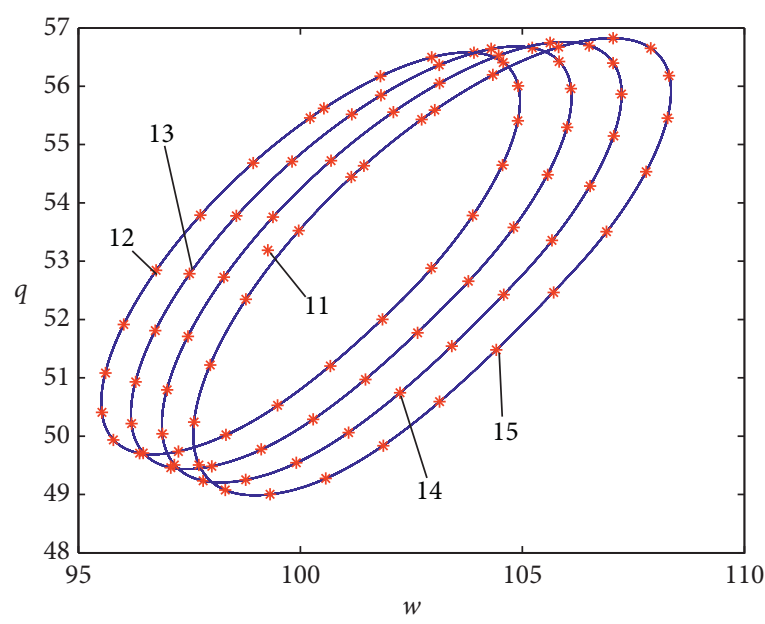

(a)

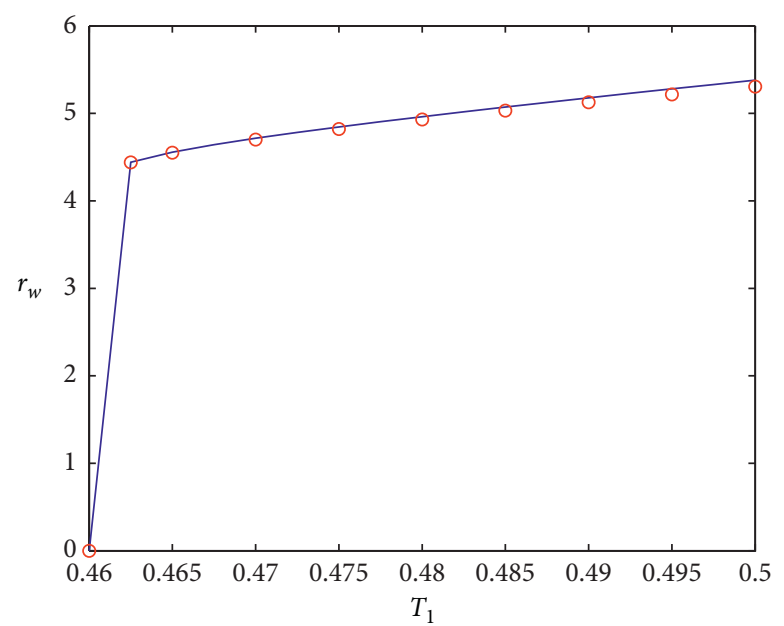

(b)

Figure 4: The phase portraits are displayed in (a) where solid blue lines are numerical results, ${ }^{*}$ results of the HB-AFT method, and $k=0.75, C=100, \alpha=0.125, \beta=0.5, \underline{b}=50, \bar{p}=0.1$, and $\bar{b}=450 . l_{1}, l_{2}, l_{3}, l_{4}$, and $l_{5}$ correspond to $T_{1}=0.46, T_{1}=0.47, T_{1}=0.48, T_{1}=$ 0.49 , and $T_{1}=0.5$, respectively. The comparisons of amplitudes of $w$ in the numerical simulations and the first-order harmonic amplitudes of $w$ by HB-AFT are presented in (b) where the solid blue line is the amplitude of numerical simulations and red ${ }^{\circ}$ are the first-order harmonic amplitudes.

TABLE 2: Results of the closed approximate periodic solutions in system (10).

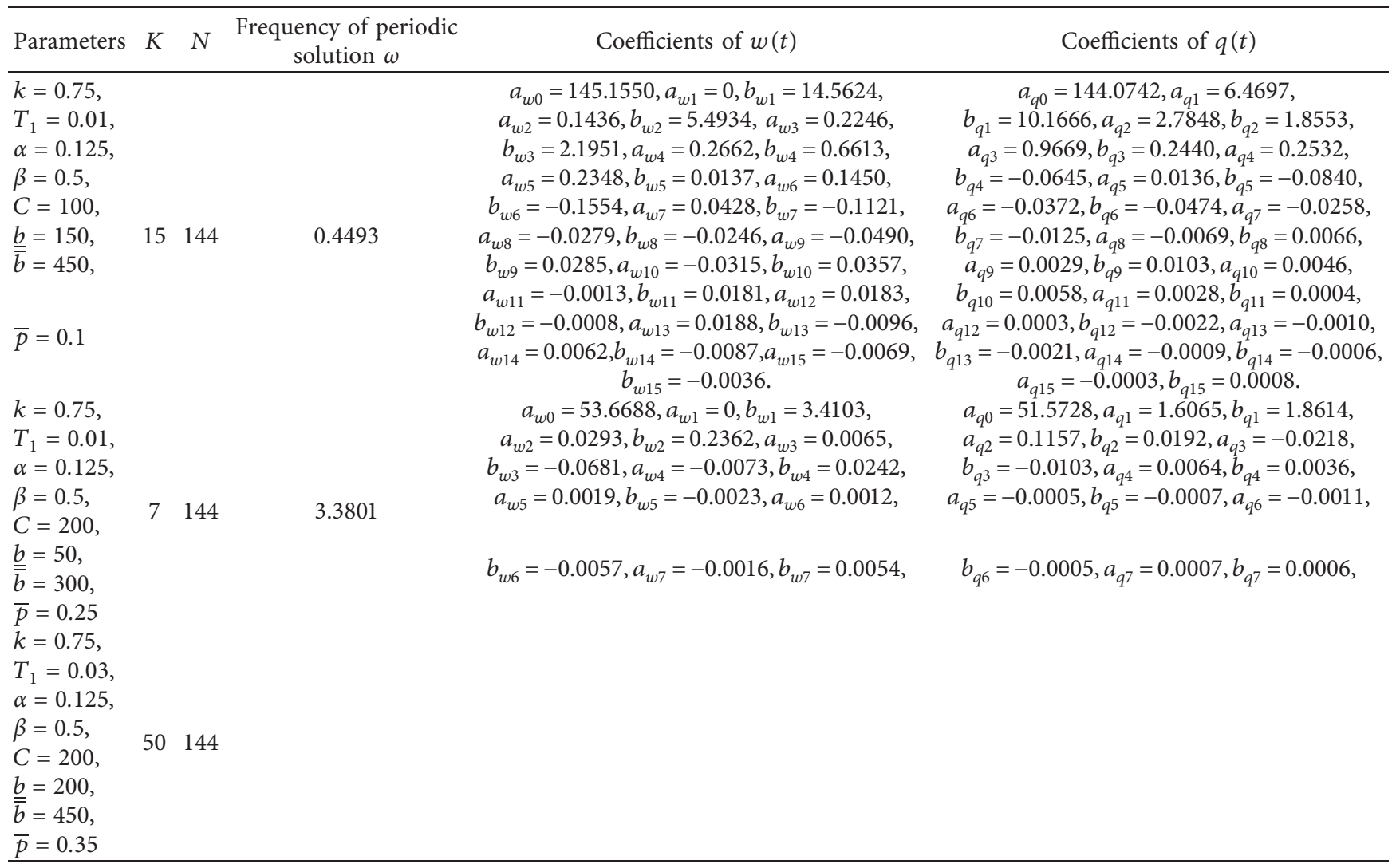

with GRED congestion control system. Here, we find a chaos with the hybrid property, and its route is very rare. So, we calculate the largest Lyapunov exponents by the small data volume method [36] to verify the chaos found in this paper.
And, we plot the bifurcation diagram generated by the Poincare section to explore the route to this chaos. The step size is 0.00002 in numerical simulation in this section. We take the following parameters: $\beta=0.5, k=0.75, \underline{b}=50, \bar{b}=450$, 


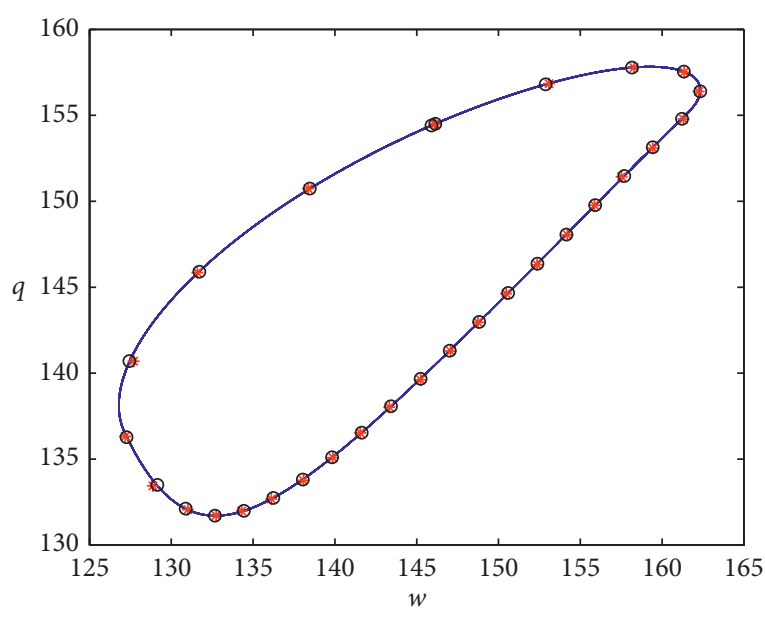

(a)

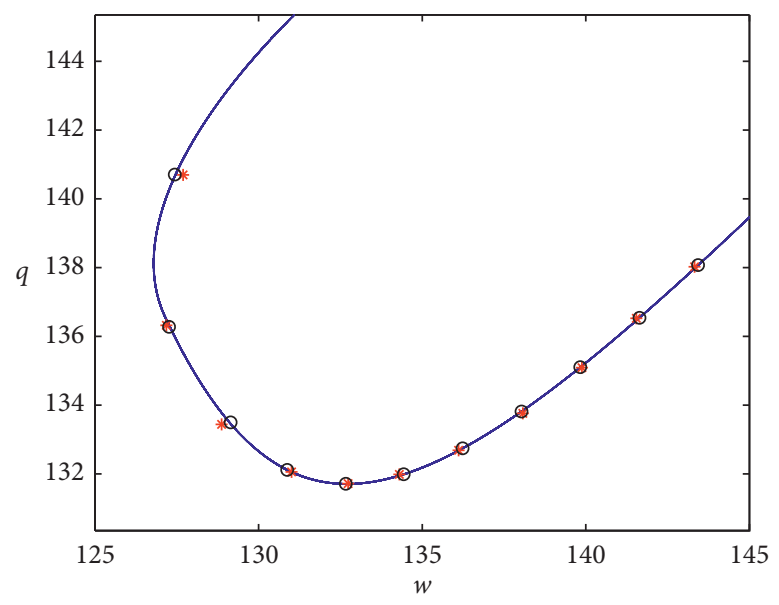

(b)

Figure 5: The phase portraits are displayed in (a) and (b), where the solid blue line represents the numerical result, red *is the result of the 6th-order HB-AFT method, and black ${ }^{\circ}$ is the result of the 15th-order HB-AFT method. (b) is the local magnification of (a).

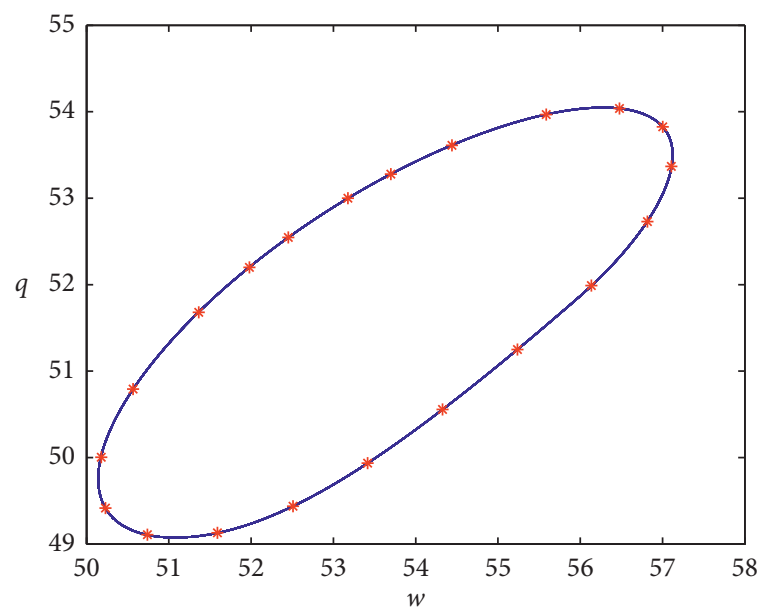

(a)

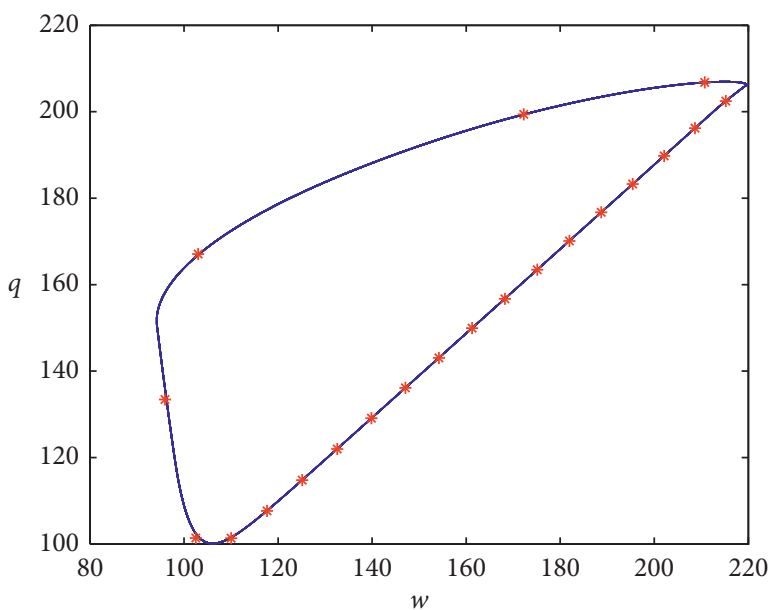

(b)

FiguRE 6: The phase portraits are displayed in (a) and (b), where solid blue lines represent numerical results and red $*$ are the results of the HB-AFT method.

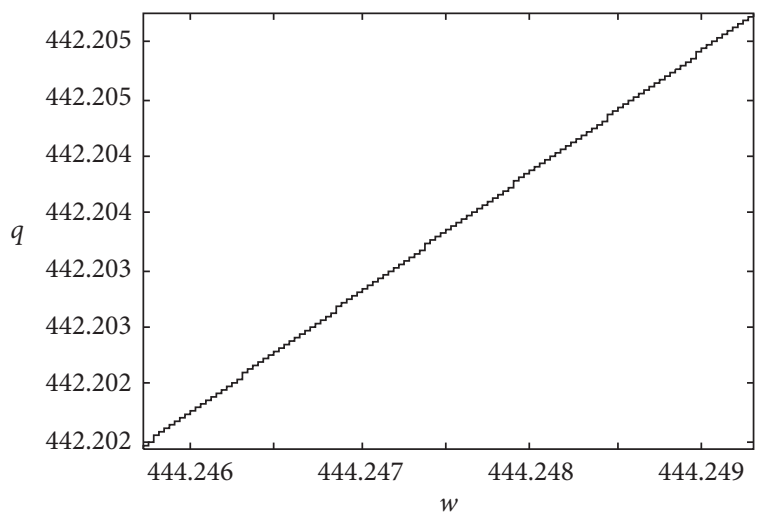

(a)

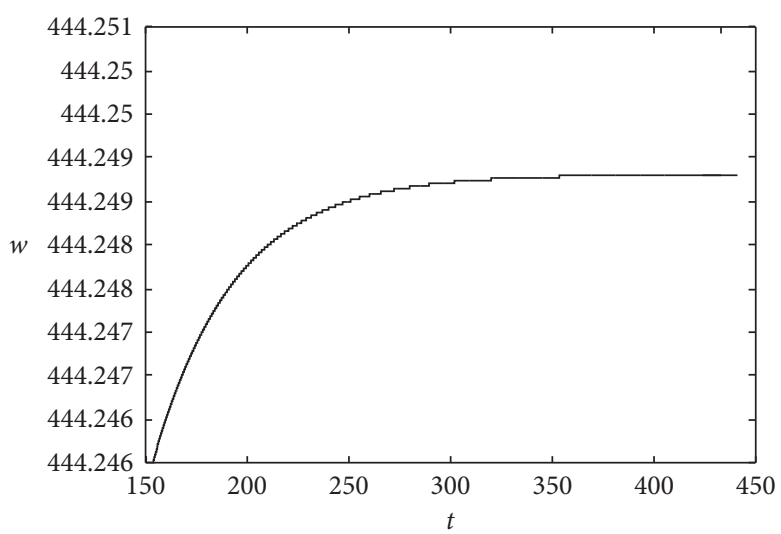

(b)

FIgURE 7: Continued. 


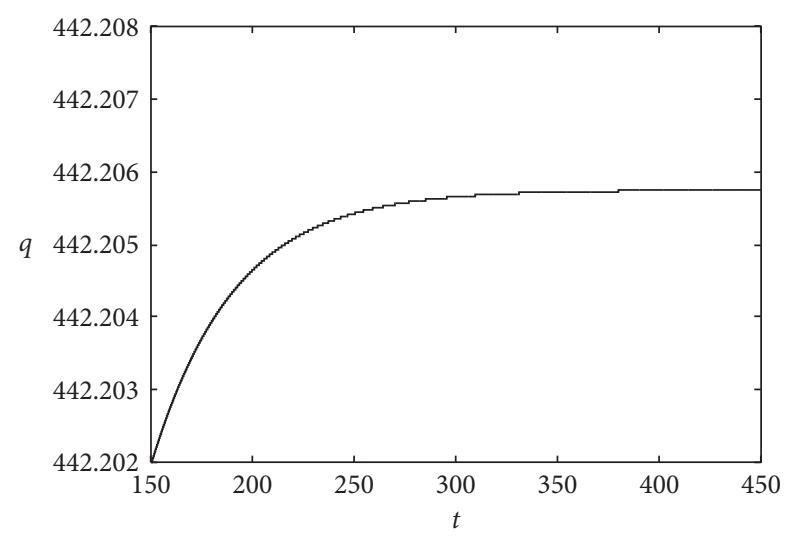

(c)

Figure 7: The stable positive equilibrium in system (10), where the phase portrait is displayed in (a) and time histories in (b) and (c).

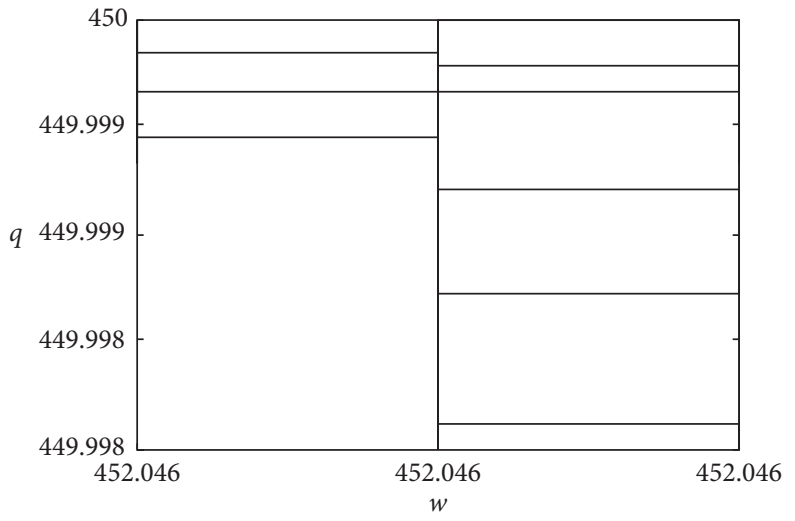

(a)

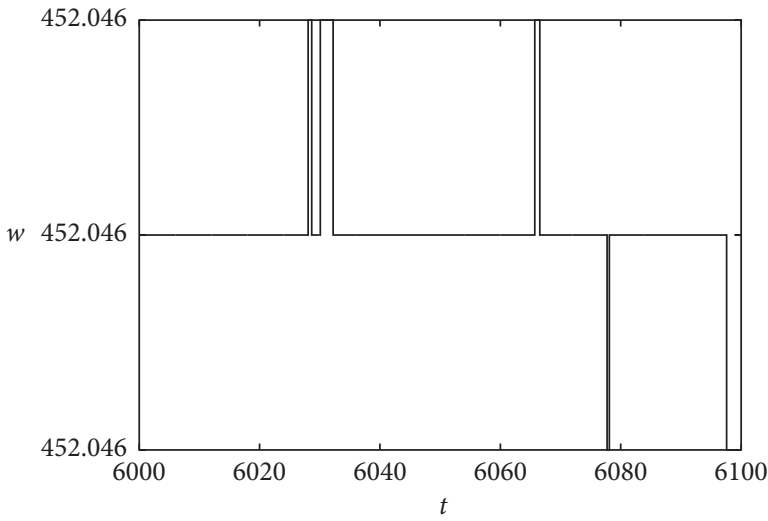

(b)

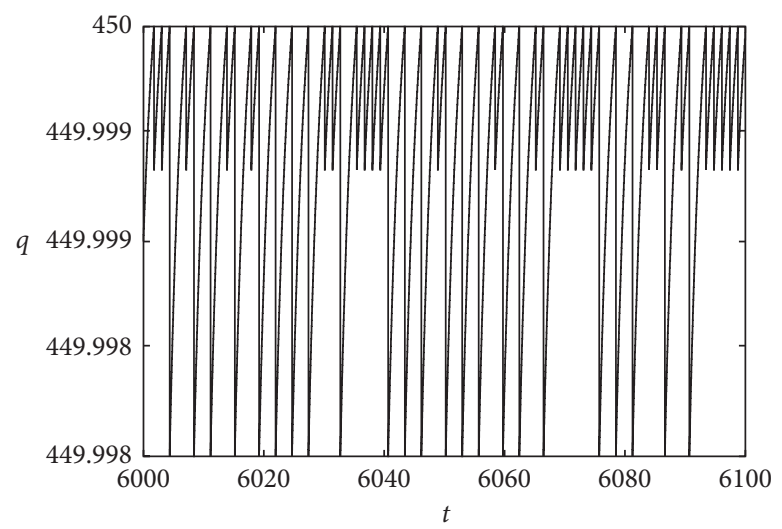

(c)

Figure 8: The irregular oscillation in system (10), where the phase portrait is in (a) and time histories in (b) and (c).

$T_{1}=0.01, C=200$, and $\bar{p}=0.0001$. We take $\alpha$ as the bifurcation parameter. For $\alpha=0.1$, there is a stable positive equilibrium $(444.249,442.206)$ in system (10). Numerical simulation is displayed in Figure 7, where the initial functions are $w(0)=444, q(0)=442, w\left(-\tau_{\max }\right)=5$, and $q\left(-\tau_{\max }\right)=5$.
For $\alpha=0.1042296$, the irregular oscillation occurs as in Figure 8 , where the initial functions are $w(0)=$ $452.045, q(0)=450.001$, and $w\left(-\tau_{\max }\right)=q\left(-\tau_{\max }\right)=5$.

Obviously $w$ and $q$ oscillate irregularly in a very small range and $q$ with a higher frequency. 


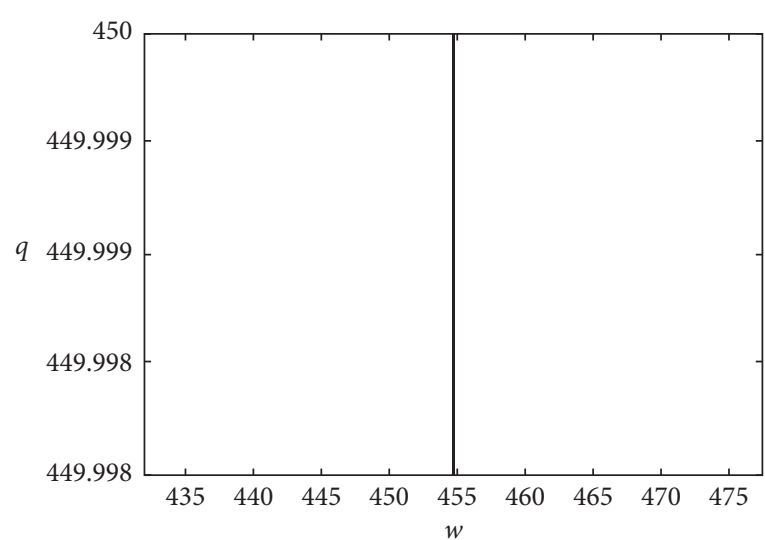

(a)

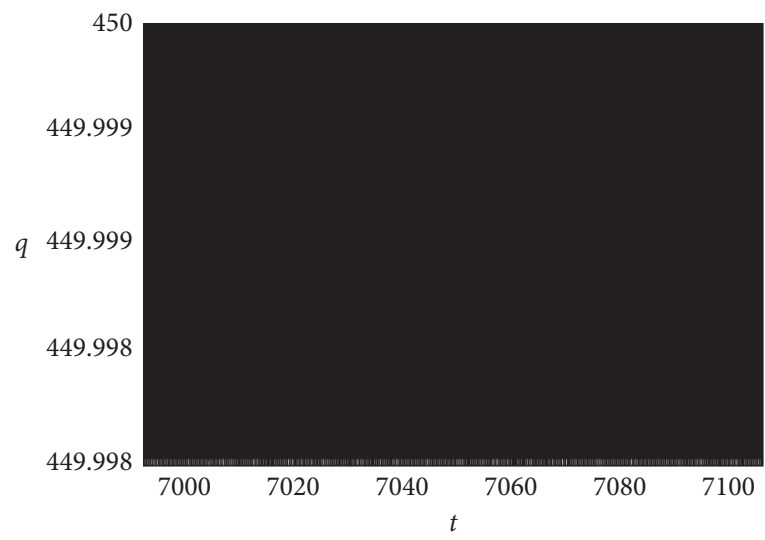

(c)

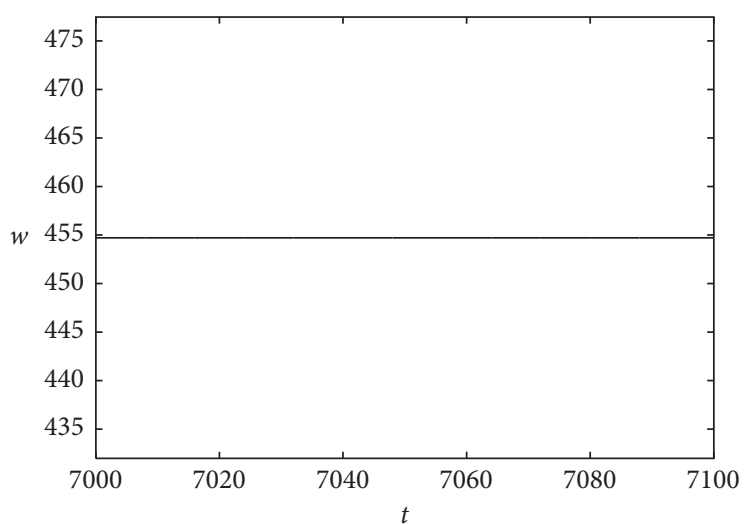

(b)



(d)

FIGURE 9: The more irregular oscillation in system (10), where the phase portrait is in (a), time histories in (b) and (c), and (d) is the local magnification of (c).

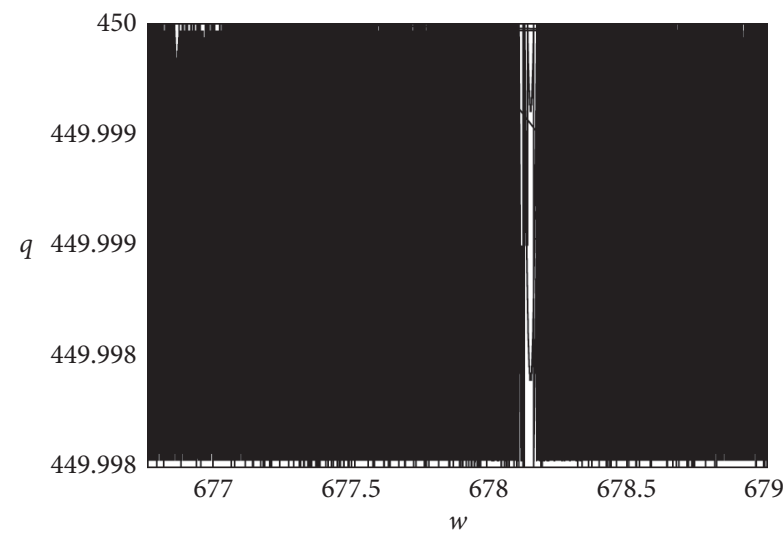

(a)

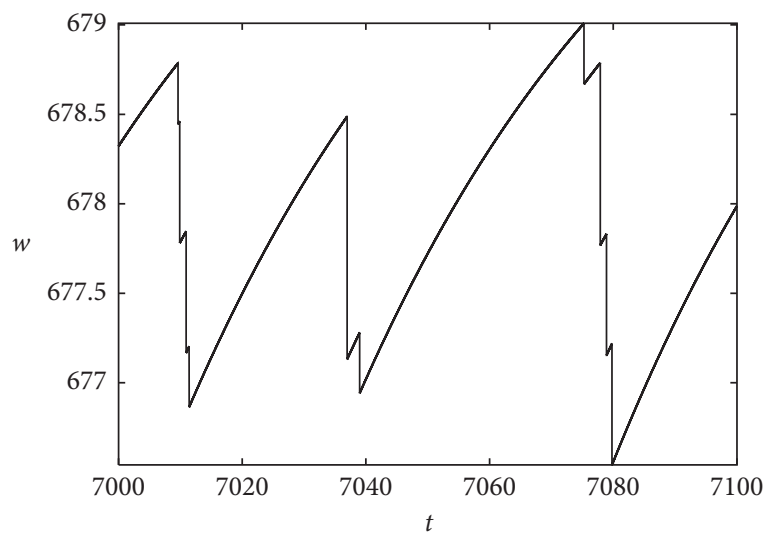

(b)

Figure 10: Continued. 


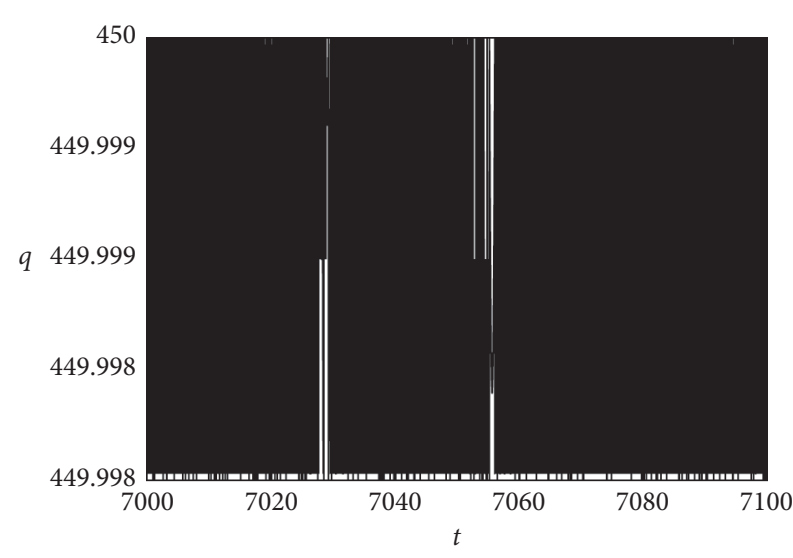

(c)

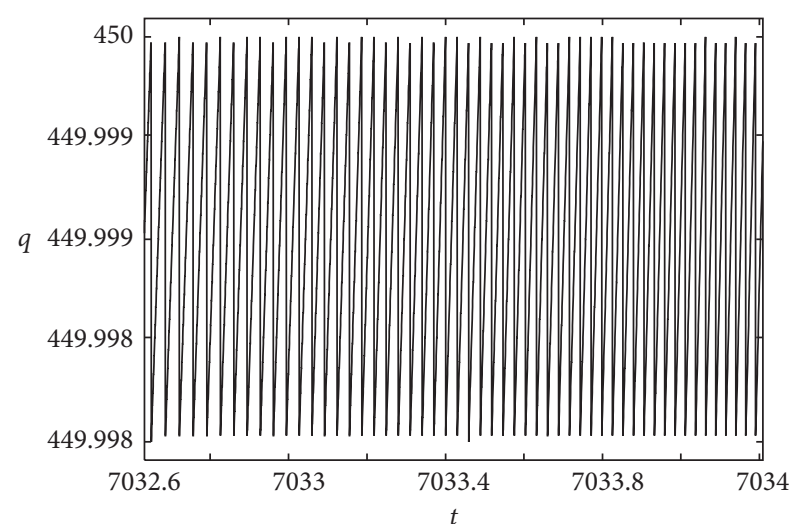

(d)

FiguRE 10: The more irregular oscillation in a broader region of system (10), where the phase portrait is in (a), time histories in (b) and (c), and (d) is the local magnification of (c).

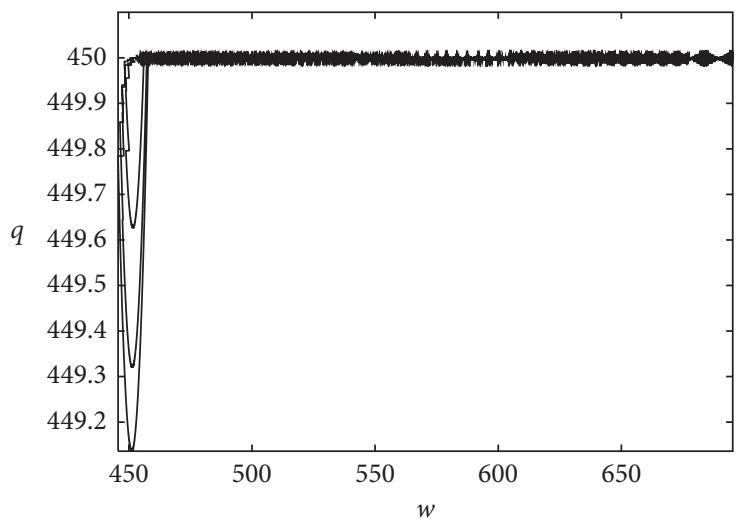

(a)

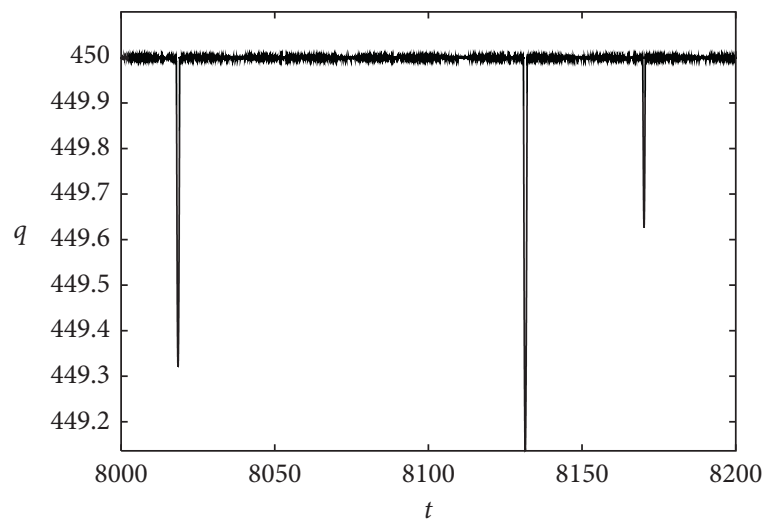

(c)

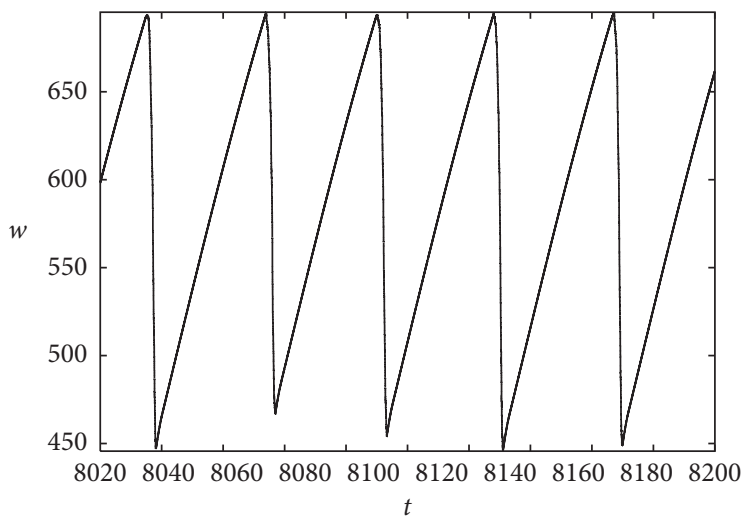

(b)

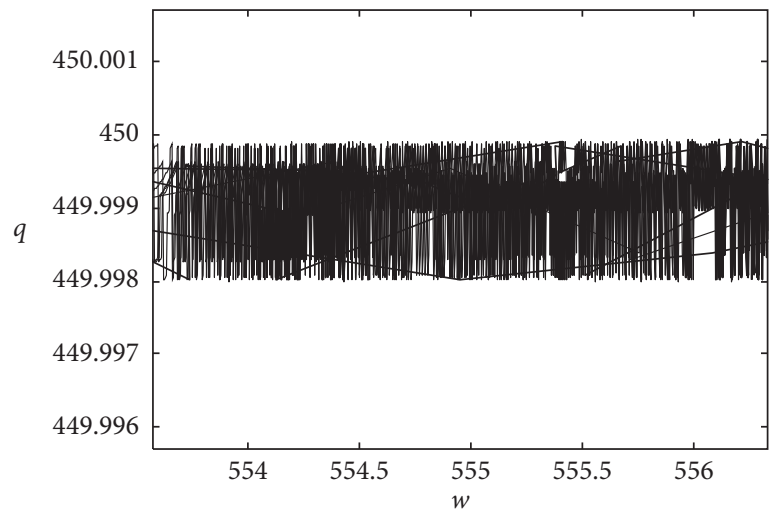

(d)

FIGURE 11: The more irregular oscillation with cycle-like part of system (10), where the phase portrait is in (a), time histories in (b) and (c), and (d) is the local magnification of the right part of (a).

When $\alpha$ continues to increase, for $\alpha=0.105$, the above oscillation becomes more irregular as in Figure 9, where the initial functions are $w(0)=452.046, q(0)=450.001$, and $w\left(-\tau_{\max }\right)=q\left(-\tau_{\max }\right)=5$. Obviously, $w$ is a constant, but $q$ oscillates irregularly in a very small range at a very high frequency. Its largest Lyapunov exponent is very large positive.

When $\alpha$ continues to increase, for $\alpha=0.174$, the irregular oscillation grows in a broader region as in Figure 10, 


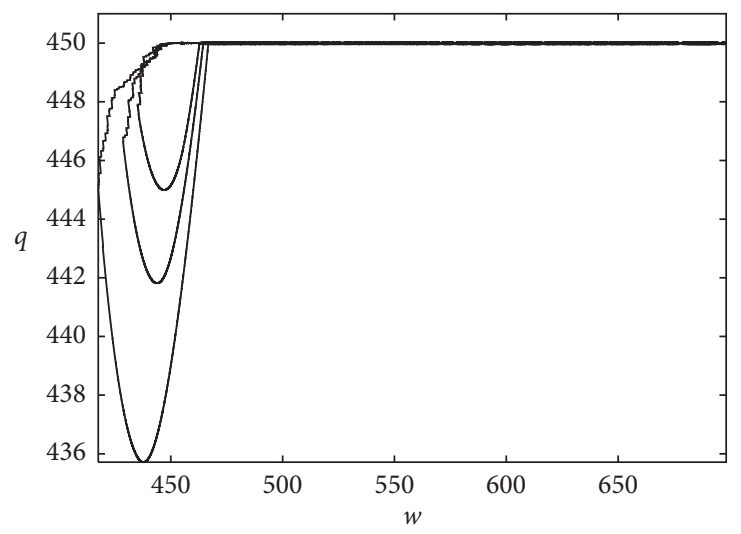

(a)

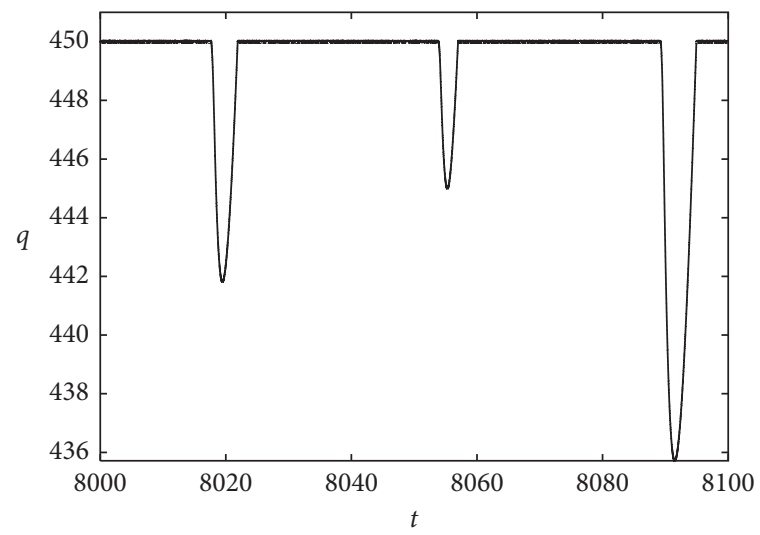

(c)

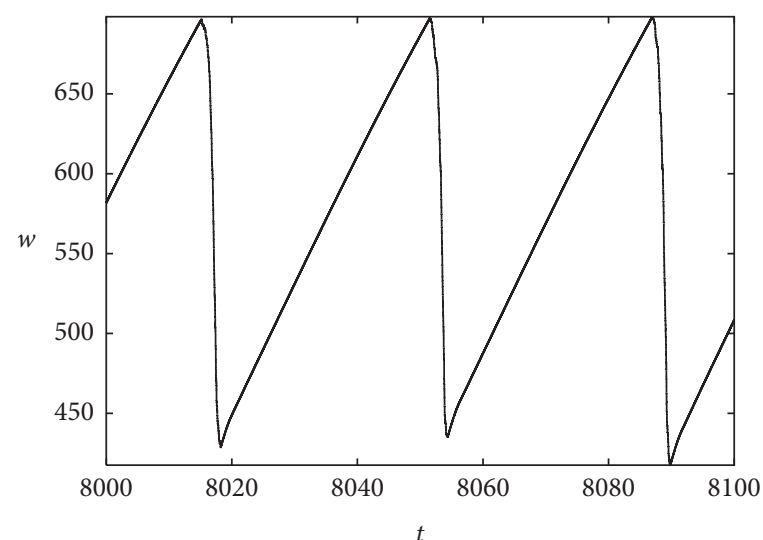

(b)

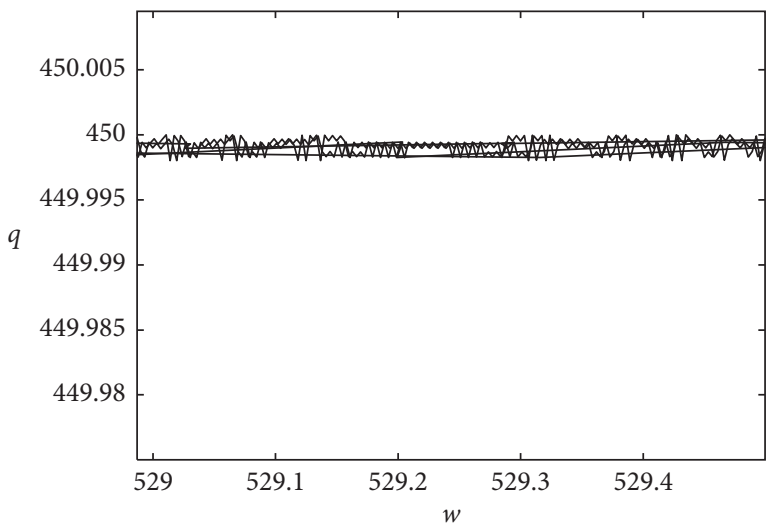

(d)

Figure 12: The chaotic oscillation with larger cycle-like part of system (10), where the phase portrait is in (a), time histories in (b) and (c), and (d) is the local magnification of the right part of (a).

where the initial functions are $w(0)=452.106$, $q(0)=450.03$, and $w\left(-\tau_{\max }\right)=q\left(-\tau_{\max }\right)=5$. $w$ moves at low frequency and large amplitude, but $q$ oscillates in a very small region and at a very high frequency. The largest Lyapunov exponent is 0.3982 .

When $\alpha$ continues to increase, for $\alpha=0.278$, the cyclelike oscillation occurring in the above irregular oscillation is displayed in Figure 11, where the initial functions are $w(0)=$ 452.195, $q(0)=450.075$, and $w\left(-\tau_{\max }\right)=q\left(-\tau_{\max }\right)=5 . w$ moves irregularly at low frequency and in large region, and $q$ still oscillates irregularly at a very high frequency but has some spikes in some small regions. The largest Lyapunov exponent is 0.2962 .

When $\alpha$ continues to increase, for $\alpha=0.3$, its cycle-like part grows larger and larger in this chaotic oscillation as in Figure 12, where the initial functions are $w(0)=$ $452.215, q(0)=450.084$, and $w\left(-\tau_{\max }\right)=q\left(-\tau_{\max }\right)=5 . w$ moves irregularly at low frequency and large region, but $q$ has some larger spikes with the increase of $\alpha$. The largest Lyapunov exponent is 0.4073 . The bifurcation diagram generated by the Poincare section in system (10) is shown in Figure 13, where $\alpha=0.1 \sim 0.3, \beta=0.5, k=0.75, \underline{b}=50, \bar{b}=$ $450, T_{1}=0.01, C=200$, and $\bar{p}=0.0001$.

In this section, according to the largest Lyapunov exponents and bifurcation diagram generated by the Poincar $\dot{e}$



Figure 13: Bifurcation diagram generated by the Poincare section of system (10), where $\alpha=0.1 \sim 0.3, \beta=0.5, k=0.75, b=50, \bar{b}=$ $450, T_{1}=0.01, C=200$, and $\bar{p}=0.0001$.

section, chaos takes place, and the route to it is a very rare route from stable equilibrium to chaos, i.e., an instantaneous and local transition from stable equilibrium to chaos or sudden transition from equilibrium to chaos [31, 32]. Specifically, system (10) has a stable equilibrium when $\alpha$ is in $[0.1,0.1042296]$ and becomes chaos from $\alpha=0.1042296$. 
$w$ and $q$ oscillate irregularly in a very small range for $\alpha$ in $[0.1042296,0.174]$, and $w$ and $q$ oscillate irregularly in a larger range for $\alpha$ in $[0.174,0.3]$.

\section{Conclusions}

In this paper, the novel state-dependent delayed nonsmooth compound TCP-GRED congestion control system is investigated fully. Firstly, we prove the uniqueness of the positive equilibrium in system (10). Then, we obtain the closed approximate periodic solutions in this state-dependent round-trip delayed nonsmooth system via the HB-AFT method. Comparisons between the results of numerical simulations and those of HB-AFT are performed. The results show that HB-AFT is simple, correct, and efficient. Finally, we find that system (10) shows complicated dynamics: chaos, perhaps it is a grazing, hybrid chaos, and the route to it is the sudden transition from stable equilibrium to chaos. Since the periodic oscillation can cause synchronisation of TCP flows and loss of throughput, further congestion and chaos induce collapse, which can hamper network performance. Therefore, to the end of stability and good performance, we should adjust the parameters carefully to avoid the periodic oscillation and chaos.

\section{Data Availability}

The data in this paper are produced by the software for nonlinear dynamics, WinPP. They can be reproduced by running WinPP with the provided parameters and initial functions by oneself.

\section{Conflicts of Interest}

The authors declare that there are no conflicts of interest regarding the publication of this paper.

\section{Acknowledgments}

The authors would like to acknowledge the financial support for this research via the National Natural Science Foundation of China (Nos. 11972327, 11372282, 11702250, and 10702065). The research and publication of this article were funded by the National Natural Science Foundation of China.

\section{References}

[1] S. Floyd, HighSpeed TCP for Large Congestion Windows, 2003, https://www.hjp.at/doc/rfc/rfc3649.html.

[2] V. Jacobson, "Congestion avoidance and control," Acm Special Interest Group on Data Communication, vol. 25, no. 1, pp. 314-329, 1988.

[3] T. Kelly and T. C. P. Scalable, "Scalable TCP," ACM SIGCOMM Computer Communication Review, vol. 33, no. 2, pp. 83-91, 2003.

[4] L. Xu, K. Harfoush, I. Rhee et al., "Binary increase congestion control (BIC) for fast long-distance networks," in Proceedings of the International Conference on Computer Communications, pp. 2514-2524, Hong Kong, China, March 2004.
[5] C. Jin, D. X. Wei, S. H. Low et al., "FAST TCP: motivation, architecture, algorithms, performance, international conference on computer communications," IEEE/ACM Transaction on Network, vol. 14, no. 6, pp. 2490-2501, 2004.

[6] K. Tan, J. Song, Q. Zhang et al., "A compound TCP approach for high-speed and long distance networks," IEEE International Conference Computer and Communications, vol. 1-7, pp. 1-12, 2006.

[7] S. Floyd and V. Jacobson, "Random early detection gateways for congestion avoidance," IEEE/ACM Transactions on Networking, vol. 1, no. 4, pp. 397-413, 1993.

[8] S. Floyd, Recommendations on using the gentle variant of RED, 2000.

[9] A. M. Y. Apreutesey, A. V. Korolkova, and D. S. Kulyabov, "Modeling RED Algorithm Modifications in the OpenModelica," in Proceedings of the Selected Papers of the IX Conference Information and Telecommunication Technologies and Mathematical Modeling of High-Tech Systems, Moscow, Russia, 2019, http://ceur-ws.org.

[10] S. Athuraliya, S. H. Low, V. H. Li, and fnm Qinghe Yin, "REM: Active queue management," IEEE Network, vol. 15, no. 3, pp. 48-53, 2001.

[11] C. V. Hollot, V. Misra, D. Towsley, and G. Weibo, "Analysis and design of controllers for AQM routers supporting TCP flows," IEEE Transactions on Automatic Control, vol. 47, no. 6, pp. 945-959, 2002.

[12] S. Manjunath and G. Raina, Analyses of Compound TCP with Random Early Detection (RED) Queue Management, Chinese Control and Decision Conference, pp. 5334-5339, IEEE, New York, NY, USA, 2015.

[13] S. Manjunath and G. Raina, "Compound TCP with random early detection (red): stability, bifurcation and performance analyses," 2019, https://arxiv.org/abs/1907.06302.

[14] D. Ding, X. Zhang, and D. Xue, "Stability analysis of internet congestion control model with compound TCP under RED," The Open Automation and Control Systems Journal, vol. 7, no. 1, pp. 1986-1992, 2015.

[15] M. D. Prokhorov and V. I. Ponomarenko, "Encryption and decryption of information in chaotic communication systems governed by delay-differential equations," Chaos, Solitons \& Fractals, vol. 35, no. 5, pp. 871-877, 2008.

[16] K. Shi, X. Liu, Y. Tang et al., "Some novel approaches on state estimation of delayed neural networks," Information Sciences, vol. 372, pp. 313-331, 2016.

[17] K. Shi, J. Wang, Y. Tang, and S. Zhong, "Reliable asynchronous sampled-data filtering of T-S fuzzy uncertain delayed neural networks with stochastic switched topologies," Fuzzy Sets and Systems, vol. 381, pp. 1-25, 2020.

[18] X. Zhang and H. Zhu, "Hopf bifurcation and chaos of a delayed finance system," Complexity, vol. 2019, Article ID 6715036, 18 pages, 2019.

[19] J. H. Shen, K. C. Lin, S. H. Chen, and K. Y. Sze, "Bifurcation and route-to-chaos analyses for Mathieu-Duffing oscillator by the incremental harmonic balance method," Nonlinear Dynamics, vol. 52, no. 4, pp. 403-414, 2008.

[20] A. Raghothama and S. Narayanan, "Bifurcation and chaos in geared rotor bearing system by incremental harmonic balance method," Journal of Sound and Vibration, vol. 226, no. 3, pp. 469-492, 1999.

[21] L. Liu and T. Kalmr-Nagy, "High-dimensional harmonic balance analysis for second-order delay-differential equations," Journal of Vibration and Control, vol. 16, no. 7-8, pp. 1189-1208, 2010. 
[22] L. Pei and L. Yang, "Prediction of Period-1 oscillations of the state-dependent delayed compound TCP model with PIE queue management policy via HDHBM," Communications in Nonlinear Science and Numerical Simulation, vol. 67, pp. 26-36, 2019.

[23] A. C. J. Luo, Regularity and Complexity in Dynamical Systems, Springer, New York, NY, USA, 2012.

[24] L. Pei and S. Wang, "Dynamics and the periodic solutions of the delayed non-smooth Internet TCP-RED congestion control system via HB-AFT," Applied Mathematics and Computation, vol. 361, pp. 689-702, 2019.

[25] Z.-y. Zhang and Y.-s. Chen, "Harmonic balance method with alternating frequency/time domain technique for nonlinear dynamical system with fractional exponential," Applied Mathematics and Mechanics, vol. 35, no. 4, pp. 423-436, 2014.

[26] S. Yamouchi, "The nonlinear vibration of flexible rotors, 1st report, development of a new analysis technique," in Proceedings of the JSME, vol. 49, no. 446, pp. 1862-1868, 1983.

[27] Y. B. Kim and S. T. Noah, "Stability and bifurcation analysis of oscillators with piecewise-linear characteristics: a general approach," Journal of Applied Mechanics, vol. 58, no. 2, pp. 545-553, 1991.

[28] Y. B. Kim and S. T. Noah, "Response and bifurcation analysis of a MDOF rotor system with a strong nonlinearity," Nonlinear Dynamics, vol. 2, no. 3, pp. 215-234, 1991.

[29] Y.-B. Kim and S. T. Noah, "Quasi-periodic response and stability analysis for a non-linear Jeffcott rotor," Journal of Sound and Vibration, vol. 190, no. 2, pp. 239-253, 1996.

[30] T.-C. Yuan, J. Yang, and L.-Q. Chen, "A harmonic balance approach with alternating frequency/time domain progress for piezoelectric mechanical systems," MECHANICAL SYSTEMS AND SIGNAL PROCESSING, vol. 120, pp. 274-289, 2019.

[31] D. J. W. Simpson, "The instantaneous local transition of a stable equilibrium to a chaotic attractor in piecewise-smooth systems of differential equations," Physics Letters A, vol. 380, no. 38, pp. 3067-3072, 2016.

[32] I. Foroni, A. Avellone, and A. Panchuk, "Sudden transition from equilibrium stability to chaotic dynamics in a cautious tâtonnement model," Chaos, Solitons \& Fractals, vol. 79, pp. 105-115, 2015.

[33] Z. T. Zhusubaliyev and E. Mosekilde, "Direct transition from a stable equilibrium to quasiperiodicity in non-smooth systems," Physics Letters A, vol. 372, no. 13, pp. 2237-2246, 2008.

[34] G. Raina, D. Towsley, D. Wischik et al., "Part II," ACM SIGCOMM Computer Communication Review, vol. 35, no. 3, pp. 79-82, 2005.

[35] G. Raina and D. Wischik, Buffer Sizes for Large Multiplexers: TCP Queueing Theory and Instability Analysis, Next Generation Internet, pp. 173-180, 2005.

[36] M. T. Rosenstein, J. J. Collins, and C. J. De Luca, “A practical method for calculating largest Lyapunov exponents from small data sets," Physica D: Nonlinear Phenomena, vol. 65, no. 1-2, pp. 117-134, 1993. 